http://jmscr.igmpublication.org/home/ ISSN (e)-2347-176x ISSN (p) 2455-0450

crossref DOI: https://dx.doi.org/10.18535/jmscr/v7i11.57

Journal Of Medical Science And Clinical Research

\title{
Study of Renal Haemodynamics Assessed by Renal Colour Doppler Ultrasound in Liver Cirrhosis
}

\author{
Authors
}

\section{Dr Tejaswi Chalumuri MD (RD), Dr Shrikant Ramahari MD (RD), Dr Y. Trinadh}

Department of Radio-Diagnosis, Andhra Medical College, Visakhapatnam, India

*Corresponding Author

\section{Dr Tejaswi Chalumuri MD (RD)}

\begin{abstract}
Background: Liver cirrhosis patients are complicated by different pathological processes leading to increased mortality and morbidity among them. Renal hemodynamic alteration leads to development of hepatorenal syndrome. Renal arterial Doppler study can be an effective screening modality for liver cirrhosis patients. Resistivity and Pusatility indices are parameters measured b renal Doppler which reflects renovascular impedance. There is good correlation between these parameters and severity of liver cirrhosis assessed by Child-Pugh scoring system.

Materials and Methods: This study included 50 cirrhotic patients divided into three groups according to Child-Pugh classification, and fifteen healthy controls. All the patients were subjected to clinical, laboratory and ultrasound evaluation to confirm the diagnosis of cirrhosis. The renal arterial duplex ultrasound examination was conducted on patients as well as controls to measure Pusatility and Resistivity index in main, segmental and cortical renal arteries.

Results: Pusatility and Resistivity index were found to be elevated in the patients of Child class $B$ and $C$ as compared to class $A$ and controls in the main and segmental renal arteries. Both indices were highest in class $C$ than $B$. The Child class $A$ and controls were not showing statistically significant difference in the main and segmental renal arteries. Resistivity index of the cortical arteries showed significant difference with that of controls. Both Pusatility and Resistivity indices were highest in class $C$ than $B$ and $A$ in cortical arteries. Oesophageal varices also showed significant correlation to elevated Pusatility and Resistivity indices in relation to Child classes.

Conclusion: Both renal Pusatility and Resistive index increase with the degree of the severity of the liver cirrhosis assessed by Child-Pugh classification. Renal duplex ultrasound is a safe, non-invasive and effective screening modality to study the renal hemodynamics and may predict renal dysfunction in different stages of the liver cirrhosis.
\end{abstract}

\section{Introduction}

Liver cirrhosis patients are complicated by different pathological processes leading to increased mortality and morbidity among them. This is in fact attributed to altered hemodynamics in cirrhosis patients. Renal hemodynamic alteration leads to development of hepatorenal syndrome. The morbidity and mortality associated with it is very high and few therapeutic options to the patients. Hepatorenal syndrome is functional form of renal failure secondary to intrinsic renal vasoconstriction ${ }^{1}$.

At present, there are no clinical or laboratory tests which are available to help in screening of such patients to detect at risk category of patients in subset of liver cirrhosis. Recently investigators 
have suggested role of cystatin $\mathrm{C}$ and exogenous excretory markers such as inulin, to monitor for development of renal dysfunction ${ }^{2,3}$. But these methods are costly and cannot be applied as screening tests for these patients.

Renal arterial Doppler study can be an effective screening modality for liver cirrhosis patients. It helps to detect intrarenal vasoconstriction associated altered renal hemodynamics due to circulatory changes in liver cirrhosis ${ }^{4}$. Resistivity and Pusatility indices are parameters measured $b$ renal Doppler which reflects renovascular impedance. There is good correlation between these parameters and severity of liver cirrhosis assessed by Child-Pugh scoring system ${ }^{5}$.

Present study was carried out to evaluate role of renal arterial Doppler to study altered renal hemodynamics in liver cirrhosis patients.

\section{Materials and Methods}

This descriptive study has been carried out in Department of Radiodiagnosis, Andhra Medical College, Visakhapatnam. A total of 50 patients of liver cirrhosis based on clinical, laboratory and ultrasonography were included in the study. The study period was from March 2017 to December 2018.

The protocol of the study was approved by the research council and ethical committee of the institute. Informed consent was taken from all patients who participated in the study.

\section{Inclusion Criteria}

1. Age group-All cirrhotic patients of age group >15years will be included.

2. Diagnosis of cirrhosis will be based on clinical, laboratory and/or ultrasound findings.

\section{Exclusion Criteria}

1. Known renal diseases such as Diabetic nephropathy, Hypertensive nephropathy other medical renal disorders.

2. Serum creatinine $>1.5 \mathrm{mg} / \mathrm{dl}$.

3. Marked hepatic encephalopathy, recent episode of gastrointestinal bleed (within 12 days).

\section{Results}

The present study focuses on two important indices of renovascular impedance namely, Pulsatality Index and Resistivity Index. These two indices were measured in three different artery levels such as main renal artery, segmental renal artery and cortical renal artery respectively. The total number of samples in the study are 65 of which the number of individuals under Child class $\mathrm{A}$ is $23(35.4 \%), \mathrm{B}$ is $9(13.8 \%), \mathrm{C}$ is $18(27.7 \%)$ and Controls $15(23.1 \%)$. The number of males over females are 55(84.4\%) and 10(15.6\%) respectively. The main objective of the study is to observe the above two indices and their average variations with respect to 3 categories of Child class. Along with this comparison summary statistics such as mean, S.D, minimum, maximum, and range are to be reported for 3 categories of Child class as well as sex.

Table 1- Child Class Distribution

\begin{tabular}{|l|c|c|}
\hline CHILD Class & Frequency & Percent \\
\hline A & 23 & 35.4 \\
\hline B & 9 & 13.8 \\
\hline C & 18 & 27.7 \\
\hline Controls & 15 & 23.1 \\
\hline Total & 65 & 100.0 \\
\hline
\end{tabular}

Figure 1- Grey Scale Features Liver Cirrosis with Ascites

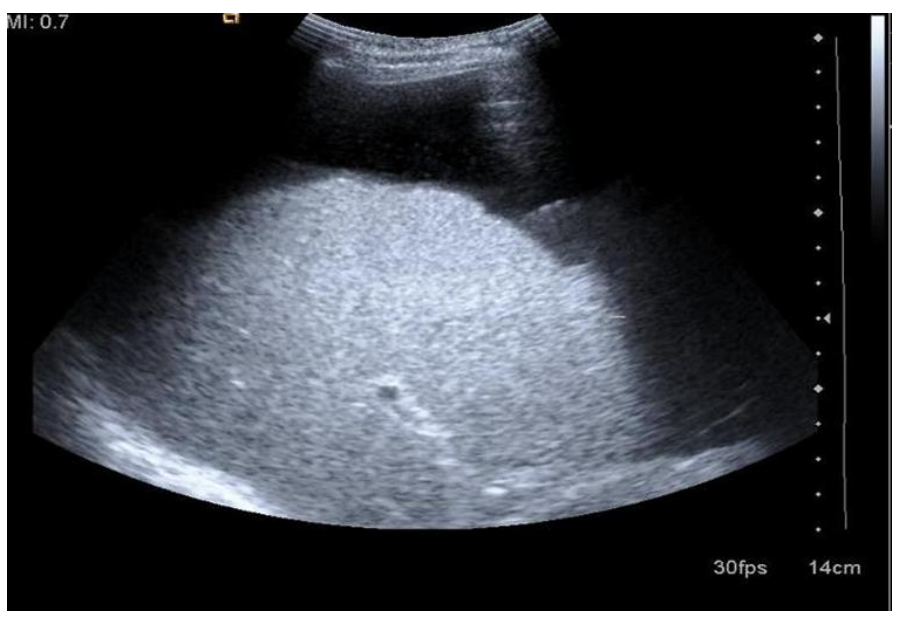


Table 2- Main Renal Artery Pulsatility and Resistivity Index

\begin{tabular}{|c|c|c|c|c|c|c|c|}
\hline & \multirow{2}{*}{ Parameters } & \multirow{2}{*}{ Statistics } & \multicolumn{4}{|c|}{ Child Class } & \multirow{2}{*}{$\begin{array}{c}\text { F-statistic } \\
\text { (p-value) }\end{array}$} \\
\hline & & & $\mathbf{A}$ & B & C & Controls & \\
\hline \multirow{10}{*}{ Main Renal Artery } & \multirow{5}{*}{$\begin{array}{l}\text { Pulsatality } \\
\text { Index }\end{array}$} & Mean & $1.042^{\mathrm{a}}$ & $1.198^{\mathrm{b}}$ & $1.439^{\mathrm{c}}$ & $1.083^{\mathrm{a}}$ & \multirow{5}{*}{$\begin{array}{l}111.430 \\
\left(0.000^{*}\right)\end{array}$} \\
\hline & & Std. Deviation & 0.062 & 0.037 & 0.110 & 0.043 & \\
\hline & & Minimum & 0.910 & 1.130 & 1.270 & 1.000 & \\
\hline & & Maximum & 1.130 & 1.260 & 1.680 & 1.140 & \\
\hline & & Range & 0.220 & 0.130 & 0.410 & 0.140 & \\
\hline & \multirow{5}{*}{$\begin{array}{l}\text { Resistivity } \\
\text { Index }\end{array}$} & Mean & $0.615^{\mathrm{a}}$ & $0.679^{b}$ & $0.751^{\mathrm{c}}$ & $0.627^{\mathrm{a}}$ & \multirow{5}{*}{$\begin{array}{c}64.980 \\
\left(0.000^{*}\right)\end{array}$} \\
\hline & & Std. Deviation & 0.034 & 0.033 & 0.038 & 0.024 & \\
\hline & & Minimum & 0.520 & 0.630 & 0.670 & 0.580 & \\
\hline & & Maximum & 0.680 & 0.720 & 0.800 & 0.660 & \\
\hline & & Range & 0.160 & 0.090 & 0.130 & 0.080 & \\
\hline
\end{tabular}

In the table 2, the statistics along with the ANOVA results are reported for 2 indices. Statistical significance was observed in both the indices $(\mathrm{P}<0.05)$ and under Pulsatility index, the Child class $\mathrm{C}$ was possessing the highest average Pulsatility Index and the least was observed in Child class A. With the aid of Multiple Comparison Test, it was clear that Child class B and $\mathrm{C}$ were differing significantly with $\mathrm{A}$ and
Controls as well as between them. Further, one fact was revealed that there was no difference between the Child class A and Controls.

The alphabets $\mathrm{a}, \mathrm{b}$ and $\mathrm{c}$ as super scripts indicates to the mean values of 3 categories of Child class. The same super scripts indicate that there is no difference between the classes and different super scripts indicates significantly different between the classes.

Figure 2- Renal Artery at Hilum Doppler

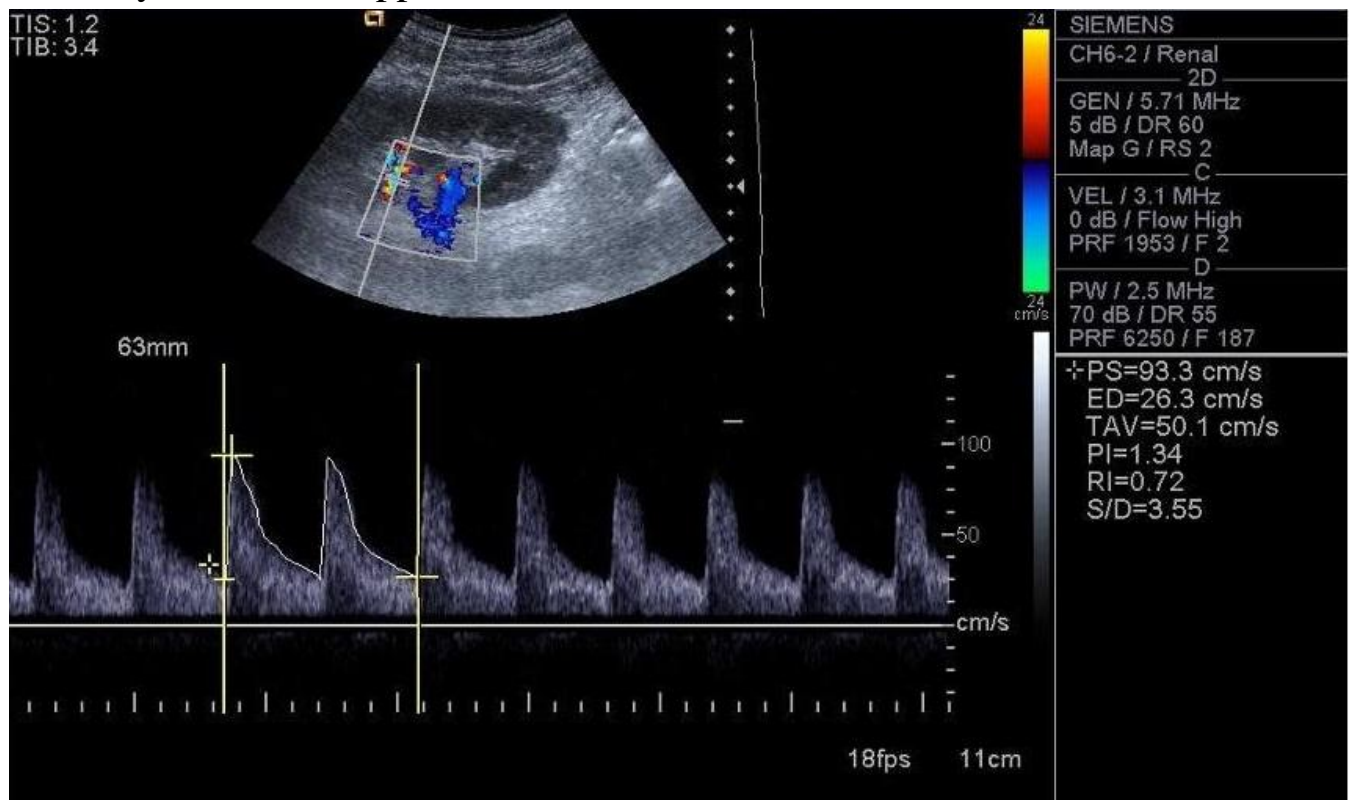

Table 3- Segmental Renal Artery

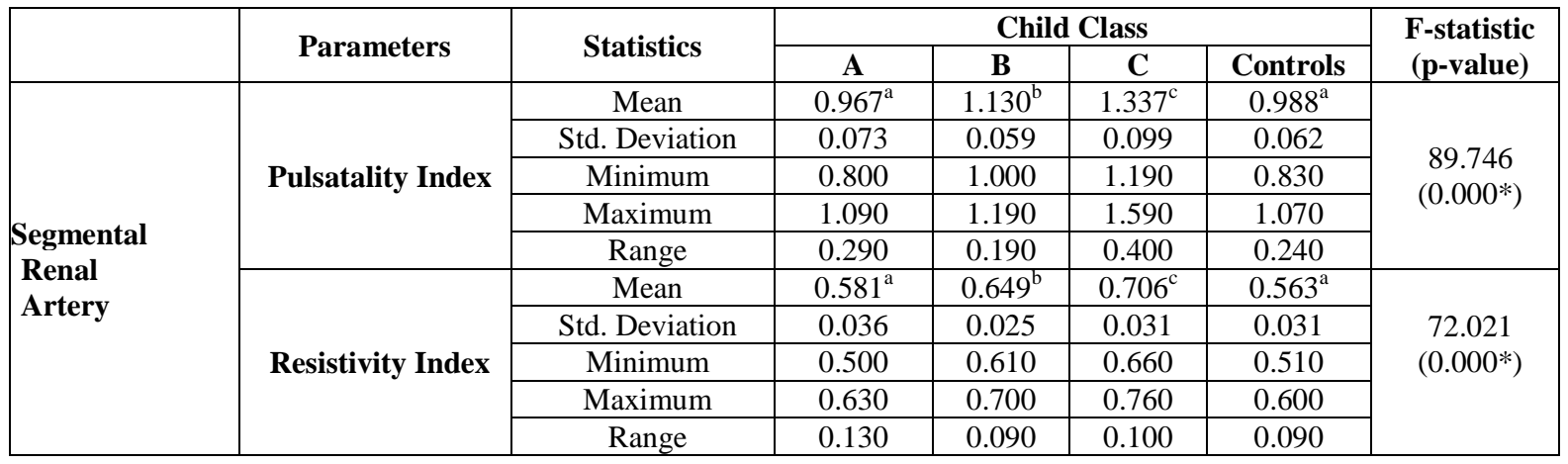


In table 3, the statistical significance was observed in both Pulsatality Index and Resistivity Index of segmental renal artery $(\mathrm{P}<0.05)$. The Child class $\mathrm{C}$ was having the highest mean, P.I and R.I, and was differing significantly with mean Pulsatility index and Resistivity index with A, B and Controls. But the Child classes A and Controls were not statistically significant

Figure 3- Segmental Renal Artery Doppler

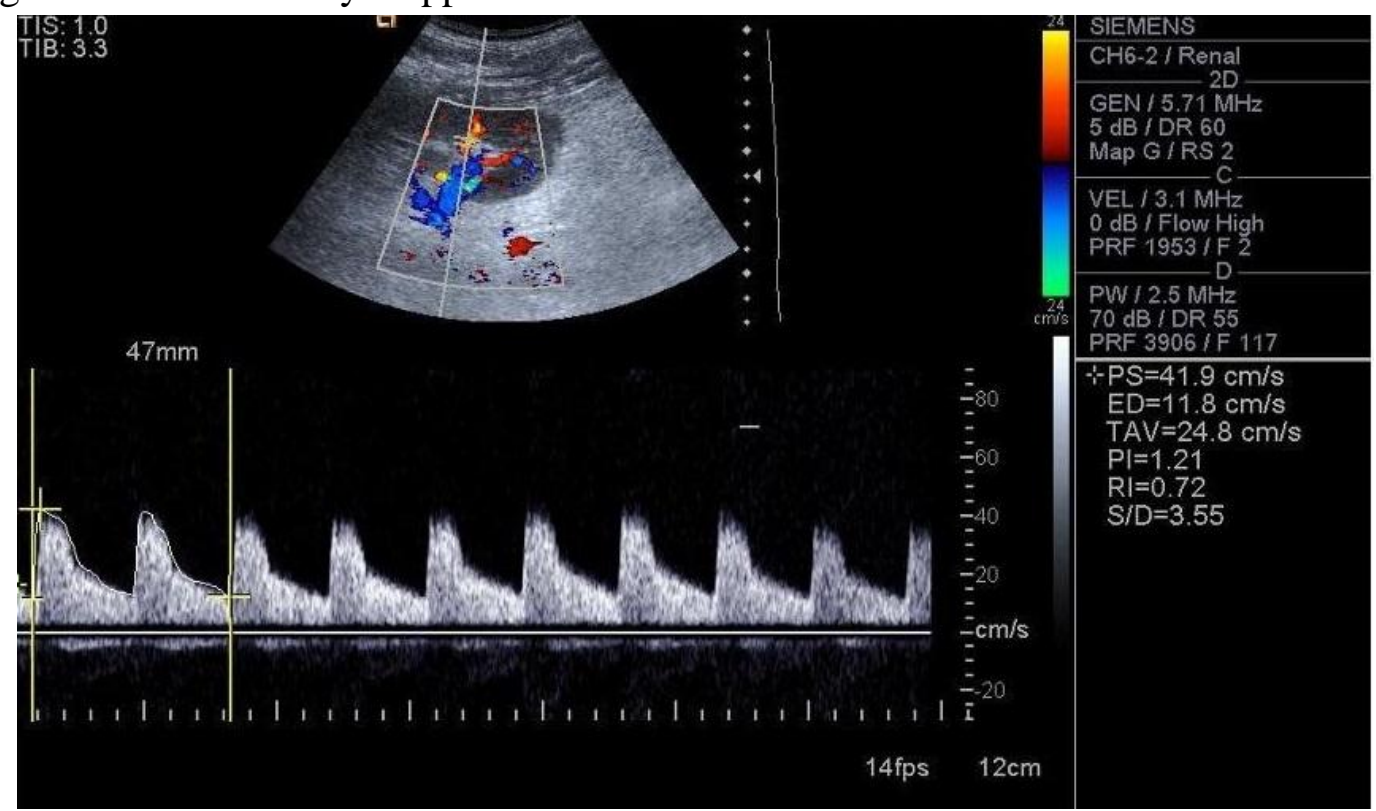

Table 4 - Cortical Renal Artery

\begin{tabular}{|c|c|c|c|c|c|c|c|}
\hline & \multirow{2}{*}{ Parameters } & \multirow{2}{*}{ Statistics } & \multicolumn{4}{|c|}{ Child Class } & \multirow{2}{*}{$\begin{array}{l}\text { F-statistic } \\
\text { (p-value) }\end{array}$} \\
\hline & & & $\mathbf{A}$ & B & $\mathbf{C}$ & Controls & \\
\hline \multirow{10}{*}{\begin{tabular}{|l} 
Cortical \\
Renal \\
Artery
\end{tabular}} & \multirow{5}{*}{$\begin{array}{l}\text { Pulsatality } \\
\text { Index }\end{array}$} & Mean & $0.890^{\mathrm{a}}$ & $1.031^{\mathrm{b}}$ & $1.234^{\mathrm{c}}$ & $0.863^{\mathrm{a}}$ & \multirow{5}{*}{$\begin{array}{c}99.409 \\
(0.000 *)\end{array}$} \\
\hline & & Std. Deviation & 0.075 & 0.067 & 0.073 & 0.068 & \\
\hline & & Minimum & 0.710 & 0.900 & 1.090 & 0.760 & \\
\hline & & Maximum & 1.000 & 1.090 & 1.330 & 0.960 & \\
\hline & & Range & 0.290 & 0.190 & 0.240 & 0.200 & \\
\hline & \multirow{5}{*}{$\begin{array}{l}\text { Resistivity } \\
\text { Index }\end{array}$} & Mean & $0.536^{\mathrm{a}}$ & $0.604^{b}$ & $0.667^{\mathrm{c}}$ & $0.498^{\mathrm{d}}$ & \multirow{5}{*}{$\begin{array}{c}81.533 \\
\left(0.000^{*}\right)\end{array}$} \\
\hline & & Std. Deviation & 0.038 & 0.037 & 0.027 & 0.033 & \\
\hline & & Minimum & 0.460 & 0.530 & 0.620 & 0.450 & \\
\hline & & Maximum & 0.600 & 0.650 & 0.720 & 0.550 & \\
\hline & & Range & 0.140 & 0.120 & 0.100 & 0.100 & \\
\hline
\end{tabular}

In table 4, statistical significance was observed in both P.I and R.I of Cortical Renal Artery $(\mathrm{P}<0.05)$. Under Pulsatality Index, the Child class $\mathrm{C}$ was differing significantly with $\mathrm{A}, \mathrm{B}$ and controls. But the child classes A and controls were statistically insignificant. An interesting fact was observed in R.I that all the categories of Child class were statistically significant and were differing to each other. Here, the Child class C possessed the highest mean Resistivity Index and the least was observed in controls. In this Resistivity Index of cortical renal artery, the Child classes $\mathrm{A}$ and Controls were also differing significantly. 
Figure 4- Cortical Renal Artery Doppler

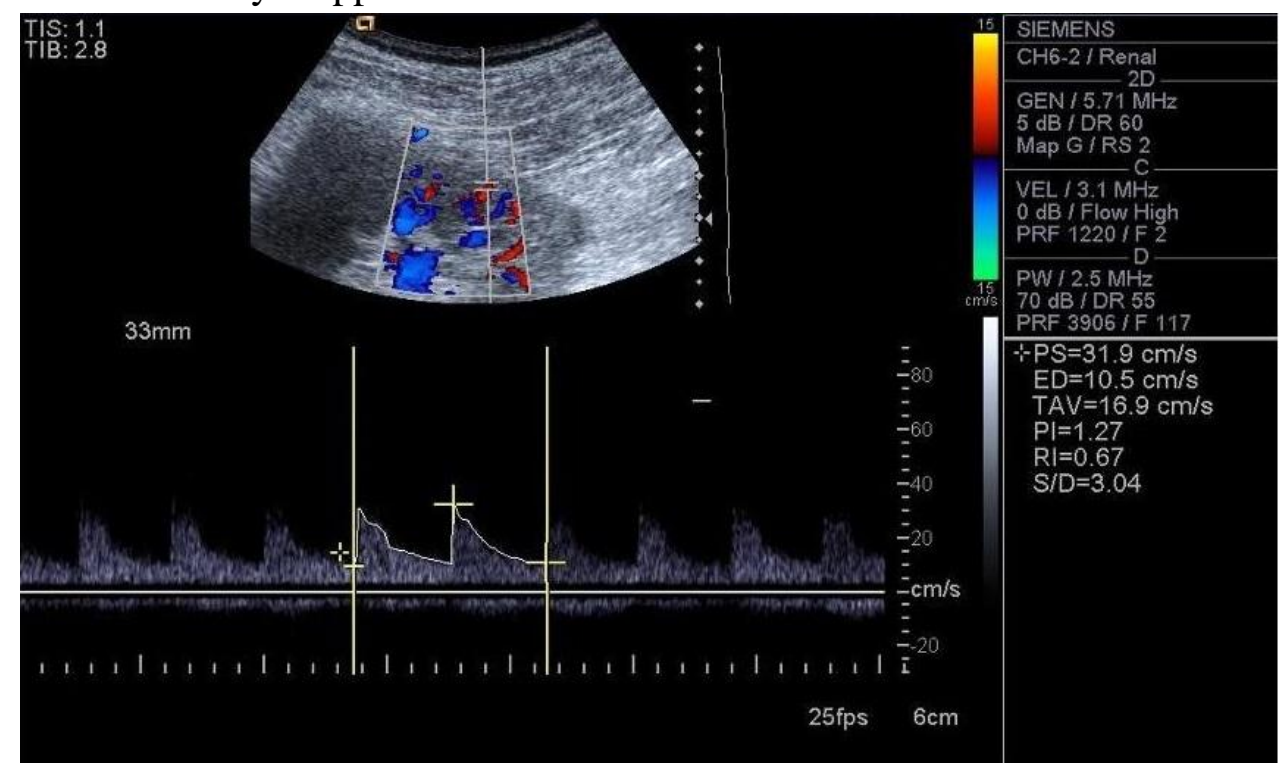

Table 5- Comparison of All Data in Male and Female

\begin{tabular}{|l|l|c|c|c|c|c|c|c|}
\hline \multicolumn{2}{|l|}{ SEX } & AGE & MRA PI & MRA CI & SRA PI & SRA RI & CRA PI & CRA RI \\
\hline \multirow{5}{*}{ Male } & Mean & 46.51 & 1.2011 & .6707 & 1.1102 & .6289 & 1.0225 & .5838 \\
\cline { 2 - 9 } & N & 55 & 55 & 55 & 55 & 55 & 55 & 55 \\
\cline { 2 - 9 } & Std. Deviation & 9.682 & 0.19133 & 0.06914 & 0.18740 & 0.06882 & 0.17308 & 0.07465 \\
\cline { 2 - 9 } & Minimum & 24 & 0.91 & 0.52 & 0.80 & 0.50 & 0.71 & 0.45 \\
\cline { 2 - 9 } & Maximum & 65 & 1.68 & 0.80 & 1.59 & 0.76 & 1.33 & 0.72 \\
\cline { 2 - 9 } & Range & 41 & 0.77 & 0.28 & 0.79 & 0.26 & 0.62 & 0.27 \\
\hline \multirow{5}{*}{ Female } & Mean & 38.30 & 1.0830 & 0.6280 & 1.0220 & 0.5760 & 0.8670 & 0.5120 \\
\cline { 2 - 9 } & N & 10 & 10 & 10 & 10 & 10 & 10 & 10 \\
\cline { 2 - 9 } & Std. Deviation & 10.955 & 0.04620 & 0.02440 & 0.04315 & 0.02591 & 0.07258 & 0.03225 \\
\cline { 2 - 9 } & Minimum & 18 & 1.00 & 0.60 & 0.94 & 0.54 & 0.76 & 0.46 \\
\cline { 2 - 9 } & Maximum & 50 & 1.14 & 0.66 & 1.07 & 0.62 & 0.96 & 0.57 \\
\cline { 2 - 9 } & Range & 32 & 0.14 & 0.06 & 0.13 & 0.08 & 0.20 & 0.11 \\
\hline
\end{tabular}

Table 5 depicts the summary information of all the parameters with respect to sex. The results show that in all the Pulsatality Index and Resistivity Index of main renal artery, segmental renal artery and cortical renal artery, the means were at higher level when compared to females. The mean age of males (46.51) was also greater than females (38.3).

Table 6- Child Class Correlation with Serum Urea and creatinine

\begin{tabular}{|l|c|c|c|c|}
\hline Parameters & Child Class & Mean & Std. Deviation & $\begin{array}{c}\text { F-value } \\
(\mathrm{p}-\text {-value })\end{array}$ \\
\hline \multirow{3}{*}{ Serum Urea } & $\mathrm{A}$ & 23.391 & 5.655 & \multirow{2}{*}{$\begin{array}{c}2.401 \\
\end{array}$} \\
\cline { 2 - 4 }$\left(0.102^{\mathrm{NS}}\right)$ \\
\cline { 2 - 4 } & $\mathrm{B}$ & 23.111 & 7.201 & \\
\hline \multirow{3}{*}{ Serum Creatinine } & $\mathrm{C}$ & 27.611 & 7.555 & \\
\cline { 2 - 4 } & $\mathrm{B}$ & 0.735 & 0.170 & 0.132 \\
\cline { 2 - 4 } & $\mathrm{B}$ & 0.667 & 0.199 & \\
\cline { 2 - 4 } & $\mathrm{C}$ & 0.828 & & \\
\hline
\end{tabular}

From above table it is clear that there is no significant correlation between Child's class and elevation of both serum urea and creatinine values.

\section{Discussion}

This prospective cross-sectional study was performed in Department of Radiodiagnosis, Department of Radiodiagnosis, Andhra Medical College, Visakhapatnam, from March 2017 to 
December 2018. This study was carried out on 50 patients of liver cirrhosis divided into three groups on the basis of Child-Pugh classification and 15 healthy controls. There were 10 from female and 55 male in present study. The age of the patients ranged from 18 to maximum of 65 years. There were 23 patients belonging to Child's class 'A', 9 to class ' $\mathrm{B}$ ' and 18 to class ' $\mathrm{C}$ '. Present study was done to observe changes in renal hemodynamics in main, segmental and cortical renal arteries in terms of elevated Pulsatility and Resistivity indices in different stages of liver cirrhosis. Early identification of these changes will be helpful to identify the nonazotemic patients who are at risk developing renal dysfunction and hepatorenal syndrome and may be beneficial in directing or modifying their the clinical management.

Hepatorenal syndrome is well recognized complication of liver cirrhosis that often develop acutely in previously nonazotemic patients. Earliest stage of apparently functional form of hepatorenal syndrome often do unrecognized because Creatinine elevation is late feature ${ }^{6}$. Hepatorenal syndrome related mortality in advanced cirrhosis is very high. Early identification of prognostic predictors remains to be topic of debate. Presently Child Pugh classification of liver cirrhosis severity is most commonly used by clinicians for classification of patients, but it has certain limitations such as subjective nature of ascites and proportionate correlation with prediction of mortality and prognosis for hepatorenal syndrome ${ }^{7}$.

Clinical manifestations and laboratory abnormality in terms of elevated urea and Creatinine are associated with very poor outcome and therefore limiting effective therapeutic outcome $^{8}$. Liver Doppler parameters such as peak systolic velocity, Resistivity and Pulsatility index of hepatic artery were not correlating with Child classification system probably due to buffer role of hepatic artery with advanced liver cirrhosis and pseudonormalisation of Doppler parameters ${ }^{9}$.

Doppler renal arterial parameters such as Resistivity and Pulsatility index measurement not only indicate

abnormal

renovascular

hemodynamic derangement in liver cirrhosis but can give information about early markers of cortical ischemia secondary to intrinsic renal vasoconstriction in patients with normal serum urea and creatinine ${ }^{10}$.

The results obtained in present study are in good agreement with the results from literature. Elevated Pulsatility and Resistivity index values are more commonly found in patients with advanced clinical stage of liver cirrhosis, but can be present in Child A and B stage also as shown by various previous studies. Platt and coworkers performed a long term follow up of 180 patients of liver cirrhosis without azotemia showing that initial elevated intrarenal Resistivity and Pulsatility index correlate with future development of renal dysfunction and hepatorenal dysfunction on their clinical follow up. They found that within 76 patients of elevated intrarenal Resistivity index more than $0.7,55 \%$ of patients developed renal dysfunction and $26 \%$ of patients developed hepatorenal syndrome whereas only $6 \%$ of patients with normal Resistivity index developed renal dysfunction ${ }^{11}$.

Our study result showed that elevated values of Pulsatility and Resistivity index in both Child group B and C in main and segmental renal artery compared to class A and controls. Cortical artery is showing elevated Pulsatility and Resistivity index in class $\mathrm{A}, \mathrm{B}$ and $\mathrm{C}$ compared to controls. We didn't follow up the patients to monitor changes of renal dysfunction or hepatorenal syndrome.

Yaseer M Fraud and co-investigators showed in their study that Pulsatility and Resistivity index values were higher for main, segmental and cortical arteries in all Child's A, B and C compared to controls. Furthermore they observed elevated values were highest in class $\mathrm{C}$ suggesting severity of ascites correlate with renovascular impedence $^{12}$. Our study showed similar results, but elevated pulsatility and Resistivity index in Child's class A were observed only in cortical artery compared to controls. We didn't find 
statistically significant difference in main and segmental renal artery Pulsatility and Resistivity index values in Child A compared to controls. This finding has not been described in available literature.

Our results are further supported by Maroto et al who demonstrated elevation of Resistivity index was significantly higher in decompensatory stage with refractory ascites than responsive ascites and compensatory stage ${ }^{13}$. Koda and their coworkers also showed that both Pulsatility and Resistivity index elevation were significantly higher in cirrhosis than controls and correlated with severity of cirrhosis assessed by Child-Pugh scoring system $^{14}$.

Rivolta et al observed in their clinical study of 57 cirrhosis patients, elevated Resistivity index correlate with severity of cirrhosis and ascites. They observed that elevated Resistivity index of cortical arteries only in Child group $\mathrm{C}$ patients with normal values in group $\mathrm{A}$ and $\mathrm{B}$ compared to controls ${ }^{15}$. On the basis of this observation, they suggested that cortical ischemia is feature of decompensated stage with refractory ascites. In our study we observed elevation of these values in cortical arteries of all the groups compared to controls, highest elevation in group $\mathrm{C}$ patients compared to $\mathrm{A}$ and $\mathrm{B}$. This is in contradiction to above observation of cortical ischemia.

Y Wang et al showed that elevated intrarenal Resistive index correlated with stage of liver cirrhosis. Their study also showed the gradient of arterial Resistivity index across main, segmental and cortical renal artery was retained in patients even at decompensatory stage with non-refractory ascites but loss of this gradient in patients of refractory ascites. So, they suggested loss of this gradient can be important and characteristic prognostic factor in development of hepatorenal syndrome $^{16}$. In our study we didn't find this observation as this gradient was retained even in patients of refractory ascites.

In our study these elevated parameters Pulsatility and Resistivity index were also showed correlation with presence of esophageal varices on upper gastrointestinal endoscopy examination. This fact was observed in Popov et al in their study 4. Colli et al in their study of 50 liver cirrhosis of Child's class A found that presence of esophageal varices correlate significantly in patients of elevated resistivity index of intrarenal artery. They suggested pathophysiological correlation between development of portal hypertension and renovascular impedance in nonascites cirrhosis patients ${ }^{17}$. Our study didn't show presence of esophageal varices on endoscopy on any of the Child's class A patients. This may be attributed to absence of significantly elevated main and segmental renal artery Pulsatility and Resistivity index in Child group A patients compared to our healthy controls.

In our study we didn't observe significant correlation between any trend of serum urea and Creatinine values with changing intrarenal resistance parameters in various stages of Child's classification of liver cirrhosis.

In this study, patients with diabetes mellitus or hypertension were excluded because of impact of these diseases on renal hemodynamics and leading to altered parameters ${ }^{18,19}$.

The following are the limitations of the present study:

1) No clinical follow up of the patients studied

2) Child's criteria was taken as sole criteria for the assessment of liver cirrhosis, which is not a perfect system due to subjective evaluation of ascites and encephalopathy ${ }^{9}$.

3) Interobserver variability of Doppler examination, which was minimized by standard protocol of renal Doppler examination.

4) Few other clinical conditions such as kidney obstruction, acute tubular necrosis, renal vein thrombosis and hemolytic uremic syndrome can affect renal resistive indices $^{6}$. This was minimized by available clinical. laboratory and imaging data of the patients. 


\section{Conclusion}

This prospective cross-sectional study was conducted in the Department of Radiodiagnosis, Andhra Medical College, Visakhapatnam. The study period was between the March 2017 to December 2018. A total of 15 controls and 50 patients of the liver cirrhosis were recruited in study based on clinical, laboratory and imaging findings, after obtaining written informed consent. The present study was done to evaluate the role of duplex ultrasound in detecting early renal hemodynamic changes seen in the liver cirrhosis patients. Pulsatility and Resistivity index were taken as parameters to assess renovascular impedance.

All 50 patients of the liver cirrhosis with normal renal profile were divided into three groups according to Child-Pugh staging system. These patients and fifteen controls underwent both grey scale and duplex ultrasound examination of renal arteries to determine Pulsatility and Resistivity indices in main, segmental and cortical renal arteries. All continuous variables were presented with its summary statistics (mean, standard deviation, range) for both patients and controls. The entire statistical analysis has been conducted with IBM SPSS 19.0 version.

The result of our study showed that changes in Pulsatility and Resistivity index evaluated using renal arterial duplex ultrasound shows good correlation with severity of the ascites and disease status assessed by Child-Pugh staging system. We found good correlation between increased renovascular impedance and presence of esophageal varices. The patients with normal renal profile and abnormal results of renal arterial duplex study can be identified before the development of clinical renal dysfunction and hepatorenal syndrome.

From our study we concluded that renal duplex ultrasound is safe and effective screening modality in liver cirrhosis patients to detect the patients who are at risk of developing renal dysfunction and hepatorenal syndrome which contribute to increased morbidity and mortality. Also, renal duplex ultrasound can be used in order to apply therapeutic interventions in these patients to reduce morbidity and mortality. Further studies are necessary to investigate the role of renal duplex ultrasound in monitoring these therapeutic interventions in liver cirrhosis patients.

\section{References}

1. Fouad YM, Mokarrab H, Elgebaly AF, ElAmin H, Abdel-Raheem EM, Sharawy MA, et al. Renal duplex Doppler ultrasound in patients with HCV related liver cirrhosis. Trop Gastroenterol Off J Dig Dis Found. 2009 Dec;30(4):213-8.

2. Ahn HS, Kim YS, Kim SG, Kim HK, Min SK, Jeong SW, et al. Cystatin C is a good predictor of hepatorenal syndrome and survival in patients with cirrhosis who have normal serum creatinine levels. Hepatogastroenterology. 2012 Jun;59 (116):1168-73.

3. Cholongitas E, Shusang V, Marelli L, Nair D, Thomas M, Patch D, et al. Review article: renal function assessment in cirrhosis - difficulties and alternative measurements. Aliment Pharmacol Ther. 2007;26(7):969-78.

4. Cardenas A, Arroyo V. Renal and circulatory dysfunction in liver cirrhosis. Pathogenesis and treatment. Ann Gastroenterol [Internet]. 2007 Mar 25 [cited 2013 Jul 28];14(3). Available from: http://www.annalsgastro.gr/index.php/anna lsgastro/article/view/81

5. Koda M, Murawaki Y, Kawasaki H. Renovascular resistance assessed by color Doppler ultrasonography in patients with chronic liver diseases. J Gastroenterol Hepatol. 2000 Dec;15(12):1424-9.

6. Schelling JR, Linas SL. Hepatorenal syndrome. Semin Nephrol. 1990 Nov;10 (6):565-70.

7. Rowe I, Neuberger J. Prognostic models in cirrhosis: an aid but not a replacement for 
clinical judgement. Liver Int. 2007;27(5):595-7.

8. Ginès A, Escorsell A, Ginès $\mathrm{P}$, Saló $\mathrm{J}$, Jiménez W, Inglada $\mathrm{L}$, et al. Incidence, predictive factors, and prognosis of the hepatorenal syndrome in cirrhosis with ascites. Gastroenterology. 1993 Jul;105(1):229-36.

9. Popov D, Krasteva R, Ivanova R, Mateva L, Krastev Z. Doppler parameters of hepatic and renal hemodynamics in patients with liver cirrhosis. Int J Nephrol. 2012;2012:961654.

10. Knapp R, Plötzeneder A, Frauscher F, Helweg G, Judmaier W, zur Nedden D, et al. Variability of Doppler parameters in the healthy kidney: an anatomic-physiologic correlation. J Ultrasound Med Off J Am Inst Ultrasound Med. 1995 Jun;14(6):4279.

11. Platt JF, Ellis JH, Rubin JM, Merion RM, Lucey MR. Renal duplex doppler ultrasonography: A noninvasive predictor of kidney dysfunction and hepatorenal failure in liver disease. Hepatology. 1994;20(2):362-9.

12. Fouad YM, Mokarrab H, Elgebaly AF, ElAmin H, Abdel-Raheem EM, Sharawy MA, et al. Renal duplex Doppler ultrasound in patients with $\mathrm{HCV}$ related liver cirrhosis. Trop Gastroenterol Off $\mathbf{J}$ Dig Dis Found. 2009 Dec;30(4):213-8.

13. Maroto A, Ginès A, Saló J, Clària J, Ginès $\mathrm{P}$, Anibarro L, et al. Diagnosis of functional kidney failure of cirrhosis with Doppler sonography: prognostic value of resistive index. Hepatol Baltim Md. 1994 Oct;20(4 Pt 1):839-44.

14. Koda M, Murawaki Y, Kawasaki H. Renovascular resistance assessed by color Doppler ultrasonography in patients with chronic liver diseases. J Gastroenterol Hepatol. 2000 Dec;15(12):1424-9.

15. Rivolta R, Maggi A, Cazzaniga M, Castagnone D, Panzeri A, Solenghi D, et al. Reduction of renal cortical blood flow assessed by doppler in cirrhotic patients with refractory ascites. Hepatology. 1998;28(5):1235-40.

16. Wang Y, Liu L-P, Bai W-Y, Wen S-B, Dan H-J, Luan Y-Y, et al. Renal haemodynamics in patients with liver cirrhosis assessed by colour ultrasonography. J Int Med Res. 2011;39(1):249-55.

17. Colli A, Fraquelli M, Pometta R, Cocciolo M, Visentin S, Conte D. Renovascular Impedance and Esophageal Varices in Patients with Child-Pugh Class A Cirrhosis1. Radiology. 2001 Jun 1;219(3):712-5.

18. Brkljacić B, Mrzljak V, Drinković I, Soldo D, Sabljar-Matovinović M, Hebrang A. Renal vascular resistance in diabetic nephropathy: duplex Doppler US evaluation. $\quad$ Radiology. 1994 Aug;192(2):549-54.

19. De Haan MW, Kroon AA, Flobbe K, Kessels AGH, Tordoir JH, van Engelshoven JMA, et al. Renovascular disease in patients with hypertension: detection with duplex ultrasound. J Hum Hypertens. 2002 Jul;16(7):501-7. 